\title{
THE MODELING OF THE EFFECT OF LUBRICANT VISCOSITY ON THE VIBRATION OF A GEAR SYSTEM
}

\author{
Wafa Krika ${ }^{1}$ - Azzedine Bouzaouit ${ }^{2 *}$
}

${ }^{1}$ Department of Mechanical Engineering, Faculty of Technology, The $20^{\text {th }}$ August 1955 University of Skikda, 21000, Algeria ${ }^{2}$ LRPCSI Laboratory of Research, Faculty of Technology, The $20^{\text {th }}$ August 1955 University of Skikda, 21000, Algeria

\begin{tabular}{l}
\hline ARTICLE INFO \\
\hline Article history: \\
Received: 15.01 .2017$. \\
Received in revised form: 12.09 .2017$. \\
Accepted: 10.10 .2017$. \\
\hline Keywords: \\
Rotating machine \\
Gear system \\
Lubricant viscosity \\
Vibration
\end{tabular}

DOI: https://doi.org/10.30765/er.38.3.1

\begin{abstract}
:
As a part of conditional maintenance, rotating machines are monitored and diagnosed in real time to increase their service life and ensure their availability in the industry. Our work aims to follow the evolution of the vibration for rotating machines through the integration of inverse problems such as degradation of the lubricant quality. Experimental tests were made in order to characterize the influence of quality lubricant (viscosity) on the vibration of a component of a rotating machine. Realized experimental bench is composed of gear device lubricated with lubricants of different viscosity grades and rotated at a variable rotational speed. In each case we measure the value of vibration. Following this experience, a vibration model has been developed depending on the change of state of lubricants, based on the results of planned experience. Further, a correlation analysis on the model obtained was realized and the results show an agreement between theory and experiment.
\end{abstract}

Though often used in industry, vibration analysis is not the best solution to secure and optimize the operation of lubricated rotating machinery; therefore, we seek other methods of surveillance. On the other hand, vibration analysis is also widely used to complement other methods, when they are not sufficient to make a diagnosis and this is particularly the case of the oil analysis that detects early stage of degradation of a lubricant, for example $[4,5,6]$.

Lubrication in the mechanical systems is the first maintenance operation essential to reduce friction, energy losses, and wear of the members in contact. $50-80 \%$ of total defects found in components for rotating machines are linked to deficient lubrication, resulting from the use of inadequate lubricant (lack or excess, aging, solid or liquid contamination). Over the course of the service, lubricants tend to 
degrade, to lose their lubricating properties due to chemical decomposition and to become contaminated by the particles caused by wearing of the parts in contact; lubricant analysis is an effective mean of monitoring [7].

From quality point of view, inadequate lubrication greatly reduces the life of the moving parts in a lubricated machine. The choice of the lubricant quality must be particularly studied [8].

In this context, our study is to monitor the RMS vibration values of a mechanical member (the case considered is a gear system) lubricated with lubricants of different viscosity grades in order to characterize the vibration behavior which can be generated by the degradation of the viscosity.

Secondly, we present a mathematical modeling by the linear regression method based on results of planned experience in order to develop a model characterizing the vibration, depending on the lubricant state change.

\section{Experimental work}

\subsection{Gear system}

Fig. 1 shows the gear device used in the experimental tests of our work. It is compound essentially of a pinion, a wheel charged by a constant load of $10 \mathrm{~kg}$, and an oil bath formed to the lower part of the toothed wheels to ensure their lubrication by splash.

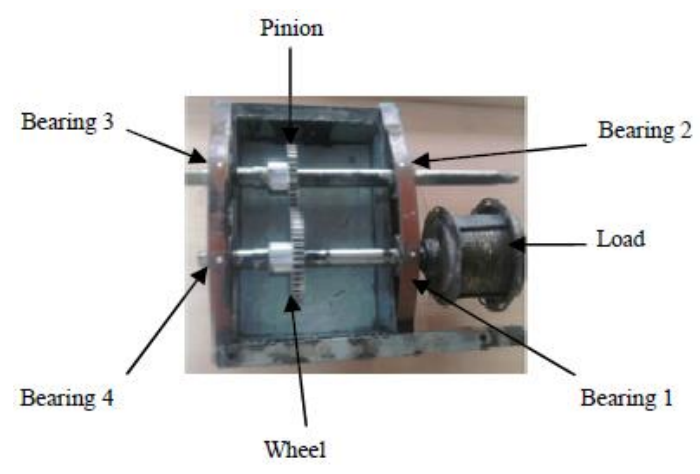

Figure 1. Gear system.

The geometrical characteristics of the gears are as follows:

The pitch diameter for pinion and wheel is respectively $76.84 \mathrm{~mm}, 135.10 \mathrm{~mm}$ and the number of teeth is 32 and 55 , with a module $2,4 \mathrm{~mm}$.

The device is driven by an electric motor in order to vary the rotational speed of the system. We have taken four measurement points which were selected on the four bearings of our gear system (P1, P2, P3 and $\mathrm{P} 4)$, two for the driving pinion and the other two for the receiving wheel, see Fig. 2.

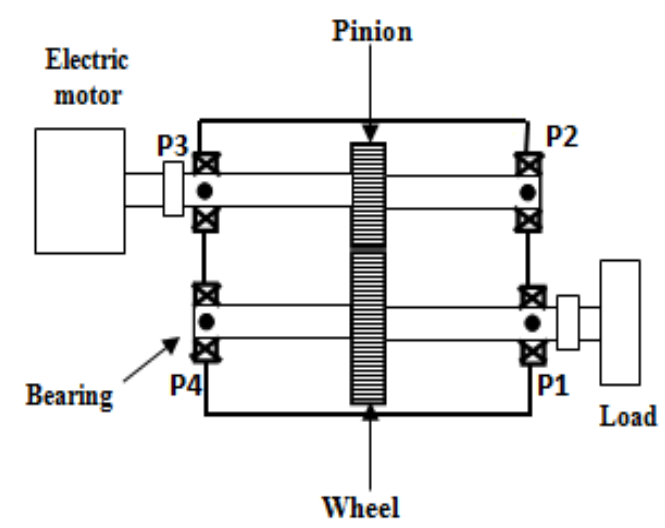

Figure 2. Experimental device gear.

\subsection{Lubricants tested}

For the lubrication of the gear system we have used six lubricants that their viscosities (V1, V2, V3, V4, V5, and V6) are given by the Table 1 .

Table 1. Characteristics of lubricants

\begin{tabular}{|c|c|c|}
\hline $\begin{array}{c}\text { Lubricant } \\
\text { codes }\end{array}$ & $\begin{array}{c}\text { Type of } \\
\text { lubricant }\end{array}$ & $\begin{array}{c}\text { Kinematic viscosity } \\
\text { at } 40{ }^{\circ} \mathrm{C} \text { in Cst }\end{array}$ \\
\hline V1 & Oil ISO 32 & 28.8 to 32.2 \\
\hline V2 & Oil ISO 68 & 61.2 to74.8 \\
\hline V3 & $\begin{array}{c}\text { Oil SAE } \\
15 W 40\end{array}$ & 105 \\
\hline V4 & EP2 grease & 150 \\
\hline V5 & $\begin{array}{c}\text { Oil SAE } \\
\text { 20W50 }\end{array}$ & 158 \\
\hline V6 & Oil SAE 90 & 215 \\
\hline
\end{tabular}

\subsection{Vibrometer "Penplus-SKF"}

For the vibration measuring we used a Vibrometer (PenPlus-SKF) shown in the Fig.3, that is a highly reliable portable instrument capable of measuring vibration caused by rotational problems. It gives the overall level RMS of the vibration velocity. 


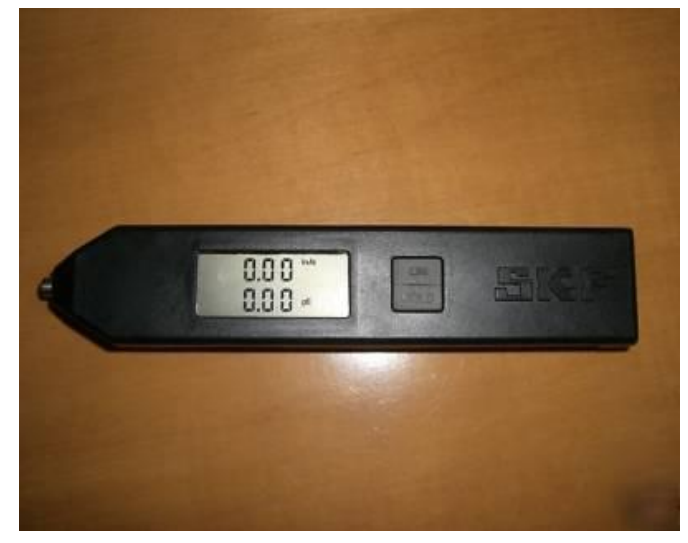

Figure 3. Photography of vibrometer Penplus SKF.

\section{Experimental results}

For each lubricant at the different rotational speeds $(\mathrm{N} 1=31.5, \mathrm{~N} 2=45, \mathrm{~N} 3=63, \mathrm{~N} 4=90, \mathrm{~N} 5=125$ and N6 = 180) RPM, we measured the (RMS) values of the vibration velocity on the four points using the vibrometer. The experiments with every lubrication condition were repeated several times to check repeatability.

\subsection{Influence of rotational speed on the vibration velocity}

Fig. 4 shows the evolution of average vibration velocity measured at points $\mathrm{P} 1, \mathrm{P} 2, \mathrm{P} 3$, and $\mathrm{P} 4$ according to the rotational speed for different viscosities tested (V1, V2, V3, V4, V5 and V6) of Table 1.

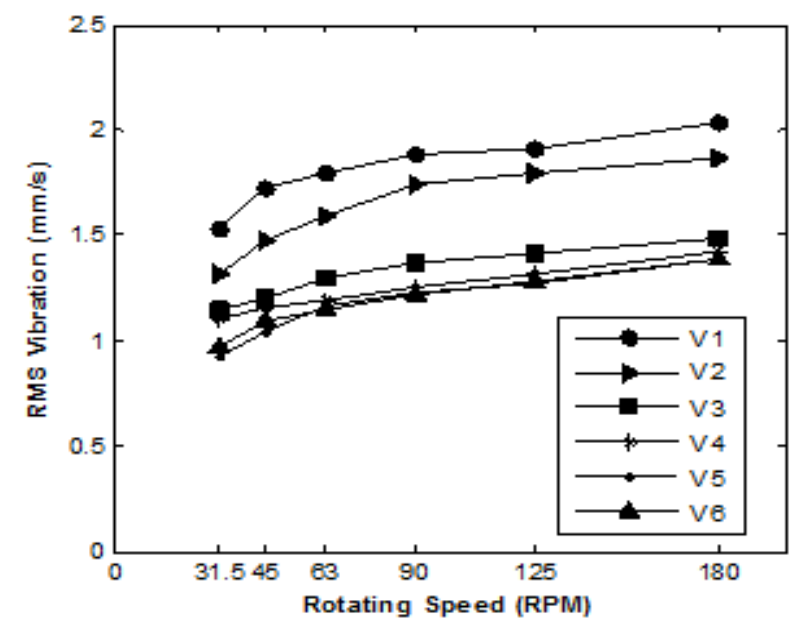

Figure 4. Evolution of the vibration velocity as a function of the rotational speed.
The vibration velocity is affected by the speed of rotation, a gradual increase in RMS values can be observed during the increase in the speed of rotation. This behavior is repeated for each lubricant.

Also, it can be observed in the case of using both lubricants (SAE 15W40 oil and EP2 grease) which have the viscosities, (V3 and V4) a slight stabilization of the vibration level of the tested system used for different speeds (the two curves have low slopes), that is to say that these viscosities are considered ideal for the lubrication of this system.

\subsection{Influence of lubricant viscosity on the vibration amplitude}

This part of our study was devoted to research and determining relationship between viscosity of a lubricant and vibration that can be produced during normal operation of the gear system.

Fig. 5 shows the variation in the average vibration velocity at different viscosities of the lubricants for each rotation speed from (N1 to N6).

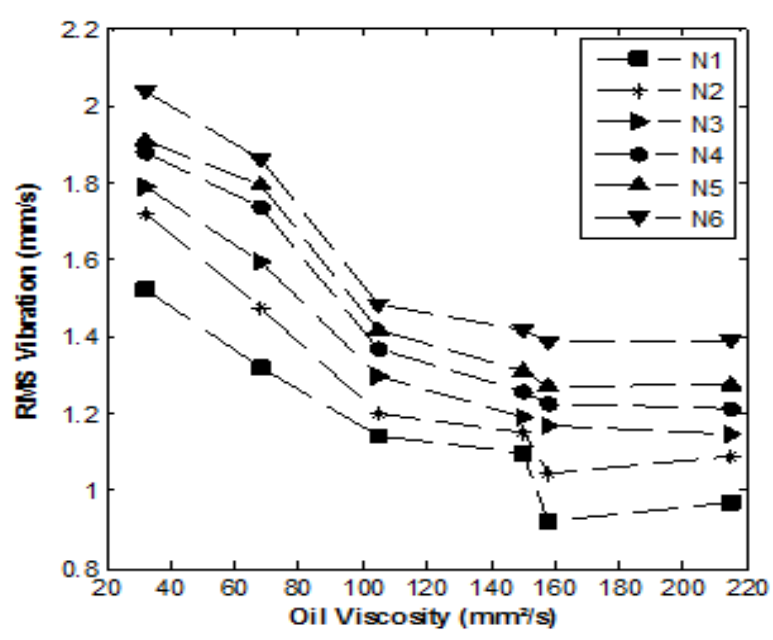

Figure 5. Variation of the vibration velocity as a function of the lubricants viscosity.

It is clear that the vibration varies inversely to the viscosity of the lubricant that is to say if the quality of the lubricant deteriorates (decrease in viscosity), the vibration level of our gear system increases accordingly.

This influence reflects the damping effect of the oil film between the surfaces in contact with its reduction increases the level of vibration. 
4 Modelling the influence of the lubricant quality on the vibration for the gear system

\subsection{The method of regression analysis and correlation}

A regression analysis seeks to determine a relationship expressing the link between so-called dependent variable and one or more so-called independent variables. The correlation is defined as a linear relationship between the two variables and the correlation coefficient measures the greater or less dependence between these two variables [9].

\subsection{The method of least squares}

In the analysis of regression and correlation, the most used method is the method of "least squares" proposed by Gauss. The principle of this method is to use $(\hat{y})$ or " $y$ valued", as opposed to $(\mathrm{y})$ which represents the " $y$ observed" [10]. We are looking for the parameters $a$ and $b$. The difference of ordinates between a point $\left(x_{i}, y_{i}\right)$ and the right point having the same abscissa is:

$$
y_{i}-\hat{y}_{i}=y_{i}-a x_{i}-b
$$

$\mathrm{y}_{\mathrm{i}}$ : Observed value.

$\hat{y}$ : Value calculated according to the model.

The linear correlation coefficient is calculated by the formula:

$$
R=\sqrt{1-\frac{\sum_{i=1}^{n}\left(\bar{y}_{i}-\hat{y}_{i}\right)^{2}}{\sum_{i=1}^{n}\left(\bar{y}_{i}-\bar{y}\right)^{2}}}
$$

The adjustment is valid for $(0.70<|R|<1)$.

\subsection{The multifactorial method}

In the general case of an optimization parameter $(y)$ of a technological process, simultaneously influencing several factors $X_{j}(j \in\{1 \div m\})$

$$
y=f\left(x_{1}, x_{2}, \ldots ., x_{m}\right)
$$

This process is described by the following model:

$$
y=C \cdot x_{1}^{b 1} \cdot x_{2}^{b 2} \ldots . x_{j}^{b j} \ldots x_{m}^{b m}
$$

The determination of $\mathrm{C}$ and $b_{j}$ is done either by the succession of unifactorial tests either by planning experiments method.

\subsection{Presentation of the model (Planning experiments)}

Linearizing equation (4) gives:

$$
\begin{gathered}
\ln y=\ln C+b_{1} \ln x_{1}+ \\
+b_{2} \ln x_{2}+\ldots+b_{j} \ln x_{j}+ \\
+b_{m} \ln x_{m} \\
\ddot{y}=b_{0}+b_{1} x_{1}^{\prime}+b_{2} x^{\prime}{ }_{2}+\ldots+b_{j} x_{j}^{\prime}+b_{m} x_{m}^{\prime}
\end{gathered}
$$

Equation (6) represents the multiple linear regression models, the regression coefficients are noted by $b_{j}$. The number of experiments to be performed is determined according to the formula:

$$
n=p^{m}
$$

Where:

$p:$ Number of level factors.

$m$ : Number of factors.

$n$ : Number of tests

For signs we give (-) and (+) for high and low levels of each factor respectively.

The range of variation of each factor is presented by the Fig. 6.

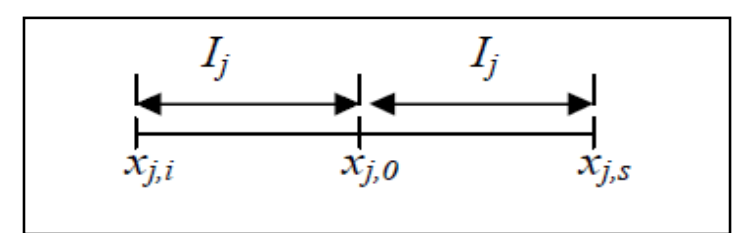

Figure 6. Basic level and the range of variation of each factor.

The codification of the factors is given by:

$$
K j=\frac{x_{j}-x_{j, 0}}{I_{j}}
$$


For model:

$$
K j=\frac{2\left(\ln x_{j}-\ln x_{j, S}\right)}{\left(\ln x_{j, S}-\ln x_{j, i}\right)}+1
$$

It is possible to gather all the elements of the experiment in a table, which is called matrix experiments [11].

For the calculation of the regression coefficients $b j$ where $(j=0: m)$ the MMC is used which gives:

$$
\begin{gathered}
b_{0}=\frac{1}{n} \sum_{i=1}^{n} Z_{0} y_{i}^{\prime} \\
b j=\frac{1}{n} \sum_{i=1}^{n} Z_{j, i} y^{\prime} i
\end{gathered}
$$

With: $j \in(1, m)$

The $Z_{0}$ quantity is a coded indication of an imaginary variable that takes only the value $(+1)$ and is used for determining the free member $b_{0}$ of the equation.

Equation (5) can also be written:

$$
\ddot{y}=b_{0}+b_{1} k_{1}+b_{2} k_{2} \ldots \ldots+b_{j} k_{j}+b_{m} k_{m}
$$

We can put the last equation in the following form:

$$
\hat{y}=e^{\ddot{y}}
$$

\subsection{Model validation}

Either the input parameters (factors) the following:

- The viscosity takes values $\left(x_{1 \min }=32\right.$ and $\left.x_{1 \max }=215\right) \mathrm{mm}^{2} / \mathrm{s}$.

- $\quad$ The rotational speed takes values $\left(x_{2 \min }=31.5\right.$ and $\left.x_{2 \max }=180\right)$ RPM.

- $y_{i}$ are the output parameters (measured vibrations).

For two input parameters, we have a model of the following form:

$$
y=C x_{1}^{b 1} \cdot C x_{2}^{b 2}
$$

We can write:

$$
\ddot{y}=b_{0}+b_{1} k_{1}+b_{2} k_{2}
$$

In the Table 2, the main parameters are grouped which establish the vibration model with $\bar{y}_{i}$ as the average vibration.

Table 2. Experimental results of vibration measurements

\begin{tabular}{|c|c|c|c|c|c|c|c|}
\hline \multirow{2}{*}{$\begin{array}{c}\text { Test } \\
\text { number }\end{array}$} & \multicolumn{2}{|c|}{ Codification of factors } & Measured vibration & \multirow{2}{*}{$\hat{y}_{i}=\ln \bar{y}_{i}$} & \multicolumn{2}{c|}{ Factor values } \\
\cline { 2 - 5 } & $\mathrm{Z}_{0}$ & $\mathrm{Z}_{1}$ & $\mathrm{Z}_{2}$ & $\bar{y}_{i}$ & & $\mathrm{X}_{1}$ & $\mathrm{X}_{2}$ \\
\hline 1 & +1 & -1 & -1 & 1.525 & 0.421 & 32 & 31.5 \\
\hline 2 & +1 & +1 & -1 & 0.970 & -0.030 & 215 & 31.5 \\
\hline 3 & +1 & -1 & +1 & 2.037 & 0.711 & 32 & 180 \\
\hline 4 & +1 & +1 & +1 & 1.391 & 0.330 & 215 & 180 \\
\hline
\end{tabular}

The calculation of the regression coefficients gives the values: $b_{0}=0.358, b_{1}=-0.208, b_{2}=0.1625$; and for the codifications $k_{l}, k_{2}$, are given by:

$$
\begin{aligned}
& k_{1}=1.049 \ln x_{1}-4.637 \\
& k_{2}=1.147 \ln x_{2}-4.957
\end{aligned}
$$

By replacing the values of regression coefficients and codifications in equation (15), we obtain:

$$
\ddot{y}=0.5169-0.218 \ln x_{1}+0.186 \ln x_{2}
$$

So the equation of vibration model will be:

$$
\hat{y}=e^{0.5169} \cdot x_{1}^{-0.218} \cdot x_{2}^{0.186}
$$

If it is assigned to the vibration velocity $V$, and $x_{1}$ and $x_{2}$ are replaced by their respective magnitudes, we have the equation for the vibration model:

$$
V=e^{0.5169} \cdot v^{-0.218} \cdot N^{0.186}
$$


Where $V:$ Vibration velocity, $\mathrm{mm} / \mathrm{s}, N$ : Rotation speed, RPM and $v$ : Kinematic viscosity, $\mathrm{mm}^{2} \mathrm{~s}^{-1}$. According to the model equation obtained, it is found that the vibration varies inversely with the viscosity; on the other hand, it is proportional with the speed of rotation.

The adjustment of this model with obtained experimental measurements is done in the following way: Consider $V_{m}$ the vibration measured and $V_{C}$ the vibration calculated by the resulting model. As well the correlation coefficient $R$ is calculated by the least square method using equation (2). The comparison between the vibration values obtained by the model and those measured for each rotation speed is presented by the Fig.7.

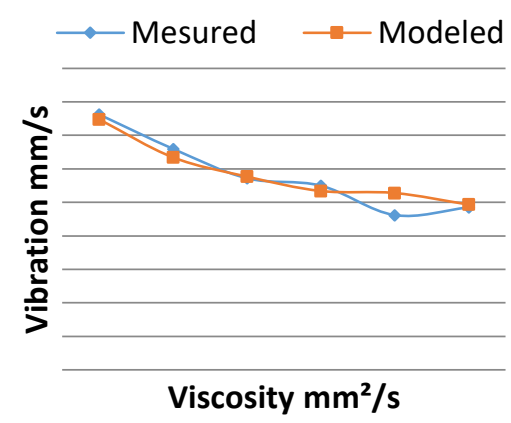

Figure 7a. Comparison between the models developed and measures for speed $N 1=31.5$ RPM.

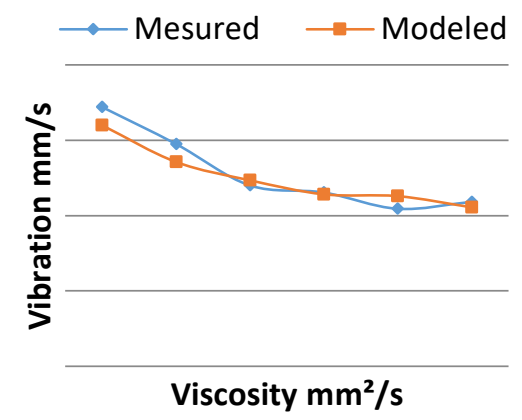

Figure 7b. Comparison between the models developed and measures for speed $N 2=45 R P M$.

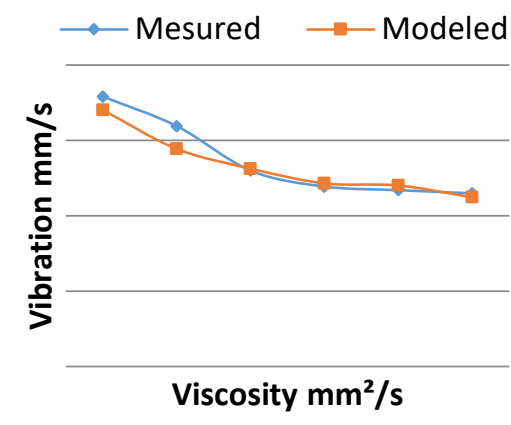

Figure $7 c$. Comparison between the models developed and measures for speed $N 3=63 R P M$.

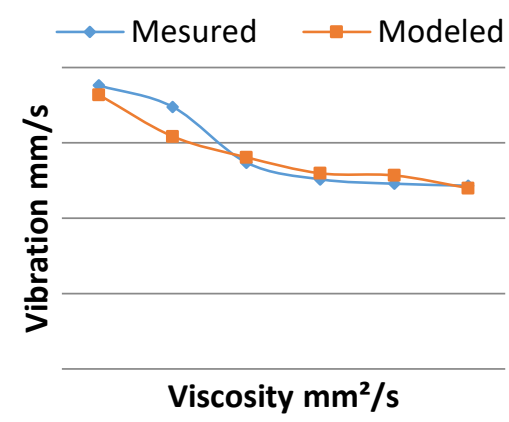

Figure $7 d$. Comparison between the models developed and measures for speed N4=90 RPM.

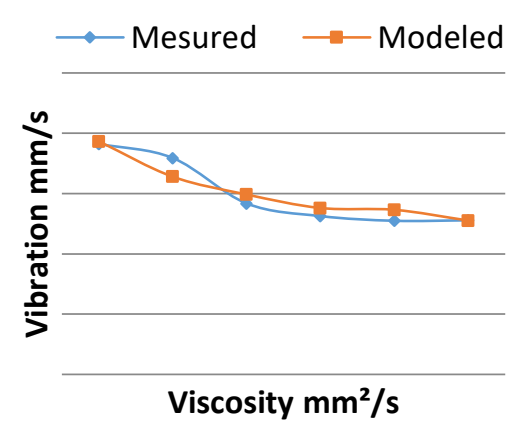

Figure 7e. Comparison between the models developed and measures for speed $N 5=125$ RPM. 


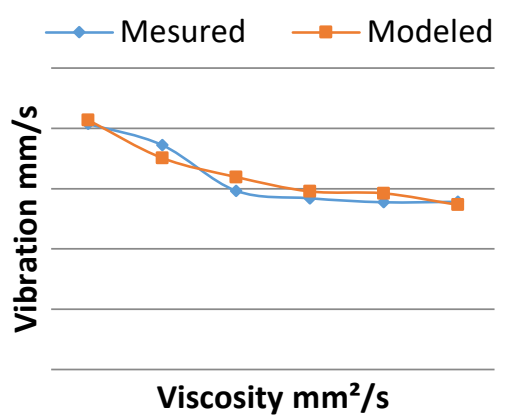

Figure 7f. Comparison between the models developed and measures for speed $N 6=180$ RPM.

In the Table 3 correlation coefficient $\mathrm{R}$ was evaluated for each case obtained.

The correlation coefficient $R$ is closer to 1 , which gives a good approximation of the variation of the vibration depending on the viscosity and the speed of rotation. We observe a good correlation between modeled and measured vibrations.

\section{Conclusion}

The main objective of the present work is to underline the importance of lubricants in the functioning of the parts for rotating machines and the choice of their quality. Based on the results obtained, it can be drawn that the vibration increases in proportion to the speed of rotation, varying inversely to the viscosity of lubricant, that is to say, the degradation of the lubricant quality (reduction in viscosity) causes the increase in the vibration level of our gear system. Therefore, the lubrication of the lightly loaded gears at average speeds, which are lubricated with relatively viscous oil, causes the separation of the teeth. This is done by a sufficiently thick film removing any metal to metal contact. Therefore, vibration in this case will be insignificant.

According to the results obtained from the regression model proposed of the vibration depending on the lubricant viscosity, it can be concluded that there is a strong linear correlation (correlation coefficient values $R \geq 0.95$ ). We see that good agreement between theory and experiment is clearly expressed.

Table 3. The calculated coefficients of correlation

\begin{tabular}{|c|c|c|c|c|c|c|}
\hline Rotation speed (RPM) & 31.5 & 45 & 63 & 90 & 125 & 180 \\
\hline Correlation coefficient $R$ & 0.9663 & 0.9745 & 0.9789 & 0.9608 & 0.9523 & 0.9605 \\
\hline
\end{tabular}

\section{References}

[1] Héng, J.: Practice of preventive maintenance, Edition Dunod, Paris, France, 2002.

[2] Bigret, R., Féron, J.: Diagnostics Maintenance availability for rotating machines: models measures - analysis of vibrations, Edition Masson Paris, 1995.

[3] Patil, S., Gaikwad, A.: Vibration Analysis of Electrical Rotating Machines using FFT. IEEE31661, 4th ICCCNT, Tiruchengode, India, 2013.

[4] Maznan, I., Izzuddin ,Z., Mohd ,I.:Condition Monitoring of Variable Speed Worm Gearbox Lubricated WithDifferent Viscosity Oils. Applied Mechanics and Materials Vols. 773774, Switzerland 2015.

[5] Lakihal, A., Hadjadj-aoul, E.: A new diagnostic strategy by conditional preventive maintenance, 4th International Conference on Computer Integrated Manufacturing CIM'2007, Algeria,03-04 November 2007.
[6] Jingwei, G., Peilin, Z., Baoyuan, L., Zhengjun, X.: An Integrated Fault Diagnosis Method of Gearboxes Using Oil Analysis and Vibration Analysis, 1-4244-1135-1/07/2007 IEEE, the Eighth International Conference on Electronic Measurement and Instruments ICEMI, 2007.

[7] Qunfeng Z., Guangneng, D., Yanling, Y., Tonghai, W,: Performance Deterioration Analysis of the Used Gear Oil, Advances in Chemical Engineering and Science, 2016, 6, 6775.

[8] Serrato, R., Maru, M., Padovese, L.: Effect of lubricant viscosity grade on mechanical vibration of roller bearings, Tribology International, 40 (2007), 1270-1275.

[9] Dodge, Y.: First steps in statistics, Edition Springer Verlag, France, 2006.

[10] Chareille, P., Pinault, Y.: Descriptive statistics, Editons Dunod, Paris, 1997. 
[11] Kamoun, A., Chaabouni, M., Ayedi, H.: Design of experiments and surface treatments, Engineering Technique (M 1428), 2001. 